\title{
Normalized Color-Ratio Modeling for CFA Interpolation
}

\author{
R. Lukac and K.N. Plataniotis
}

\begin{abstract}
A normalized color-ratio model suitable for color filter array (CFA) interpolation schemes in singlesensor imaging devices is introduced. The first proposed solution utilizes linear shifting of the CFA inputs, whereas the second design uses both scaling and shifting operations to normalize color components appearing in the CFA interpolator's input. The utilization of the proposed models can significantly boost the performance of most well-known CFA interpolators. Experimental results indicate that the CFA solutions employing the proposed models exhibit superior performance and eliminates color moire, aliasing and color shifts in the full color camera output. ${ }^{l}$
\end{abstract}

Index Terms - Single-sensor imaging, digital camera, camera image processing, Bayer pattern, color filter array interpolation, demosaicking, data normalization.

\section{INTRODUCTION}

Single-sensor imaging devices (Fig. 1) use a single charged couple device (CCD) or a complementary metal oxide semiconductor (CMOS) sensor with a color filter array (CFA) to produce a two-dimensional array or mosaic of color components. Such a CFA image is a low-resolution color image due to fact that only a single spectral component is available at each spatial location. Using the Red-Green-Blue (RGB) Bayer CFA pattern (Fig. 2) [1], the restored, highresolution RGB color image output is obtained by interpolating the missing two color components from the spatially adjacent CFA data [2]. This process is known as CFA interpolation [3],[4] or demosaicking [5],[6],[7] and is an integral part of cost-effective single-sensor devices such as image-enabled wireless phones, pocket-size imaging devices and imaging devices for surveillance and automotive applications.

Demosaicking methods rely on color models to complete the interpolation process. Popular schemes such as the smooth hue transition (SHT) interpolation scheme [8], the Kimmel's algorithm (KA) [9], and the saturation based adaptive interpolation (SAI) scheme [10] employ the color-ratio model [8],[11]. Note that other camera image processing steps such as CFA image zooming [12] and demosaicked image postprocessing [13] employed in the digital camera pipeline can use the color-ratio model as well. The color-ratio model utilizes both the spectral and spatial characteristics of the RGB

\footnotetext{
${ }^{1}$ Manuscript received March 25, 2004.

The authors are with The Edward S. Rogers Sr. Department of ECE, University of Toronto, Canada.

Corresponding Author: Dr. Rastislav Lukac, Bell Canada Multimedia Laboratory, Room BA 4157, The Edward S. Rogers Sr. Department of ECE, University of Toronto, 10 King's College Road, Toronto, Ontario, M5S 3G4, Canada (e-mail: lukacr@ieee.org)
}

Contributed Paper

Manuscript received March 25, 2004

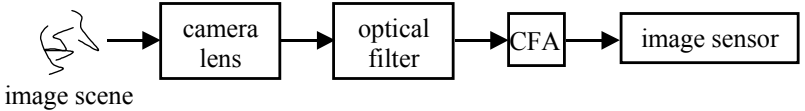

Fig. 1. A single-sensor imaging device.

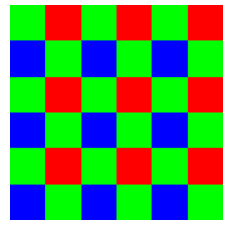

Fig. 2. Bayer CFA pattern [1].

image and is used to interpolate the missing color components using the neighboring color vectors and the available color component positioned at an interpolation location.

The rest of this paper is organized as follows. In Section II, the fundamentals of Bayer CFA image demosaicking based on a conventional color-ratio model are introduced. The proposed two normalized color-ratio models suitable for any CFA based interpolation operations performed in the digital camera pipeline are introduced in Section III. Motivation and design characteristics are discussed in detail. In Section IV, the demosaicking schemes (SHT, KA, SAI) operating on the proposed models are tested using a variety of color images. Finally, conclusions are drawn in Section V.

\section{DEMOSAICKING USING COLOR-RATIO MODEL}

Let us consider, a $K_{1} \times K_{2}$ gray-scale image $z: Z^{2} \rightarrow Z$ representing a two-dimensional matrix of integer samples $z_{(r, s)}$ with $r=1,2, \ldots, K_{1}$ denoting the image row and $s=1,2, \ldots, K_{2}$ indicating the image column, acquired by a CCD or a CMOS sensor. Since the sensor is essentially a monochromatic device being only capable of obtaining a single measurement of luminance per spatial location, a CFA is used to separate incoming light into a specific spatial arrangement of color components. Although many CFA can be used in the camera image pipeline [13], the three-color RGB Bayer CFA pattern [2] is the most commonly used due to the simplicity of the subsequent demosaicking procedure. In the Bayer CFA pattern, half of the pixels $z_{(r, s)}$ correspond to the $\mathrm{G}$ components, whereas the $\mathrm{R}$ and $\mathrm{B}$ components are assigned the other half of the pixels. Using a Bayer CFA pattern with a GRGR phase in the first row (Fig. 2) [13],[14],[15], the sensor values form a mosaic of Red (R), Green (G), and Blue (B) color components. Thus, gray-scale pixels $z_{(r, s)}$ can be transformed into the RGB vectors $\mathbf{x}_{(r, s)}=\left[x_{(r, s) 1}, x_{(r, s) 2}, x_{(r, s) 3}\right]$ with $x_{(r, s) k}$ indicating the $\mathrm{R}(k=1), \mathrm{G}(k=2)$ and $\mathrm{B}(k=3)$ component, as follows [16]: 


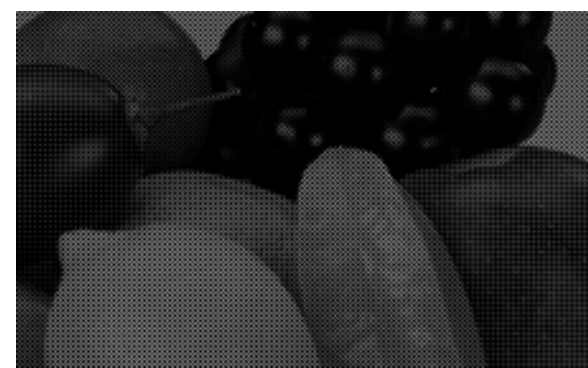

(a)

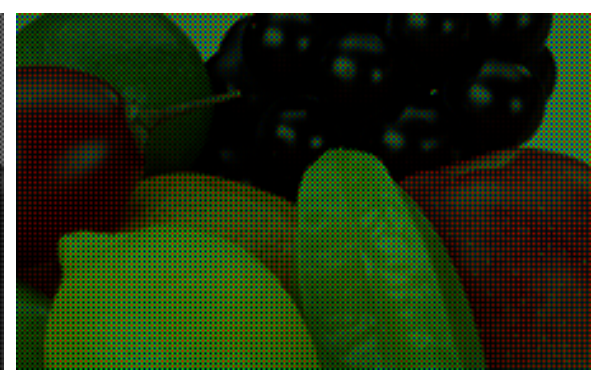

(b)

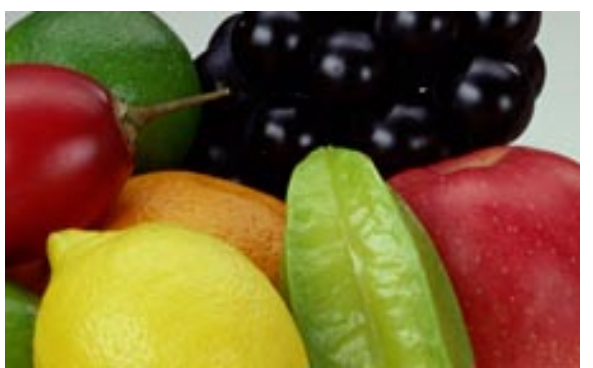

(c)

Fig. 3. Image scenario represented as: (a) a gray-scale Bayer CFA image, (b) a color Bayer CFA image, (c) a full-color image captured using a three-sensor imaging device.

$\mathbf{x}_{(r, s)}= \begin{cases}{\left[z_{(r, s)}, 0,0\right]} & \text { for } r \text { odd and } s \text { even, } \\ {\left[0,0, z_{(r, s)}\right]} & \text { for } r \text { even and } s \text { odd } \\ {\left[0, z_{(r, s)}, 0\right]} & \text { otherwise. }\end{cases}$

resulting in a $K_{1} \times K_{2}$ RGB image $\mathbf{x}: Z^{2} \rightarrow Z^{3}$. This color image represents a two-dimensional matrix of three-component samples. Since only a single measurement $x_{(r, s) k}$ varying in $k$ is available in each spatial location $(r, s)$, color vectors $\mathbf{x}_{(r, s)}$ are completed using two zero components.

Due to the double sampling frequency of the $G$ components Bayer CFA demosaicking algorithms start the interpolation procedure with the $\mathrm{G}$ color plane. In order to proportionally represent the contribution of the adjacent color components most, demosaicking algorithms determine the missing $\mathrm{G}$ color component $x_{(r, s) 2}$ using neighboring $\mathrm{G}$ components as follows [16]:

$x_{(r, s) 2}=\sum_{(i, j) \in \varsigma} w_{(i, j)}^{\prime} x_{(i, j) 2}$

where $x_{(i, j) 2}$ denotes the $\mathrm{G}$ component of the color vectors $\mathbf{x}_{(i, j)}=\left[x_{(i, j) 1}, x_{(i, j) 2}, x_{(i, j) 3}\right]$, with $(i, j) \in \varsigma$, denoting spatial location arrangements on the image lattice. Assuming the $\mathrm{G}$ component interpolators used in the well-known KA and SAI demosaicking solutions, $\zeta=\{(r-1, s),(r, s-1),(r, s+1),(r+1, s)\}$ describes a diamond-shape arrangement shown in Fig. 4a.

Each input component $x_{(i, j) 2}$ is associated with a nonnegative normalized weighted coefficients $w_{(i, j)}^{\prime}$ defined as follows [9],[16]:

$$
w_{(i, j)}^{\prime}=\frac{w_{(i, j)}}{\sum_{(g, j) \in \varsigma} w_{(g, h)}}
$$

where $w_{(i, j)}$ is a non-negative weight reflecting the edge sensitivity of the demosaicking solution. Using some form of inverse gradients [4],[9],[10],[16] the edge-sensing weights $w_{(i, j)}$ are used i) to regulate the contribution of the available color components inside the spatial arrangements described by $\varsigma$, ii) to emphasize inputs which are not positioned across an edge, and iii) to direct the interpolation process along the edges. Since the obtained large gradients correspond to the directions defined across the edge, inversely proportional values appropriately penalize the associated inputs [16]. Since both KA [9] and SAI [10] schemes uses an edge-sensing mechanism, which is well-known in camera image processing community, we will focus on the problems with the color-ratio model in these schemes rather to repeat the description of weights' calculations. Note that the SHT scheme [8] is a nonadaptive interpolation scheme which ignores the edge information and thus, it uses fixed weights set to the identical number, most often to $w_{(i, j)}=1$.

The use of (2) completes the G color plane of the image $\mathbf{x}$. Since the $\mathrm{G}$ component is available in each spatial location $(r, s)$, the arrangement of the color components used in (1) should be redefined as [7],[13]:

$\mathbf{x}_{(r, s)}= \begin{cases}{\left[x_{(r, s) 1}, x_{(r, s) 2}, 0\right]} & \text { for } r \text { odd and } s \text { even, } \\ {\left[0, x_{(r, s) 2}, x_{(r, s) 3}\right]} & \text { for } r \text { even and } s \text { odd } \\ {\left[0, x_{(r, s) 2}, 0\right]} & \text { otherwise. }\end{cases}$

reflecting the fact that in $\mathrm{R}$ or $\mathrm{B}$ CFA locations two color components are now available. Thus, both $\mathrm{RG}$ or $\mathrm{BG}$ components can be used in the subsequent demosaicking steps to interpolate the missing $\mathrm{R}$ or $\mathrm{B}$ components in the positions corresponding to original G CFA locations.

It should be mentioned that natural images exhibit a high spectral correlation among color channels and thus, the utilization of the spectral correlation that exits between $\mathrm{R}$ and $\mathrm{G}$ (or $\mathrm{B}$ and $\mathrm{G}$ ) color bands allows to use more information from the color image $\mathbf{x}$ during demosaicking. Thus, the demosaicking schemes reduce color artifacts in the demosaicked images. The schemes here under consideration, namely SHT, KA and SAI, uses the color-ratio model of [8] to take the information from the different spectral bands.

\section{A. Color-ratio model based CFA interpolator}

The color-ratio model is based on the assumption of hue uniformity enforced through $\mathrm{R} / \mathrm{G}$ and $\mathrm{B} / \mathrm{G}$ ratios in localized image areas [8],[11]. Thus, two RGB vectors $\mathbf{x}_{(r, s)}$ and $\mathbf{x}_{(i, j)}$ 
(a)
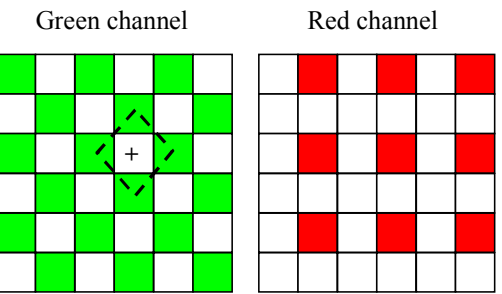

Blue channel

(b)
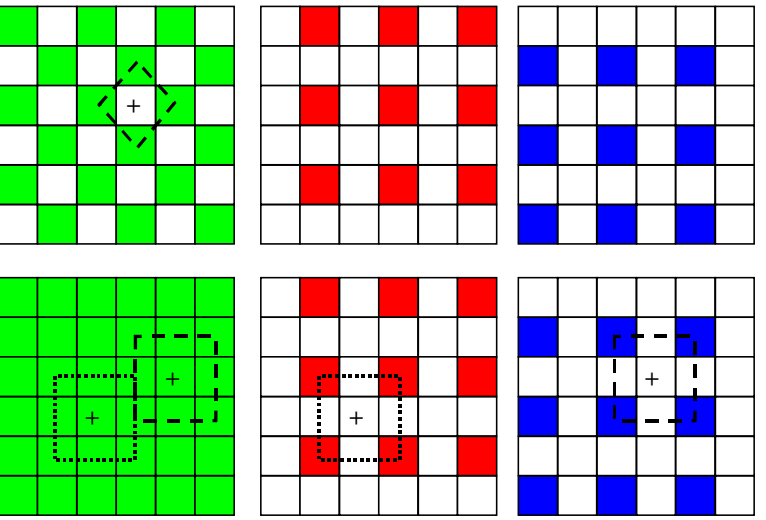

(c)
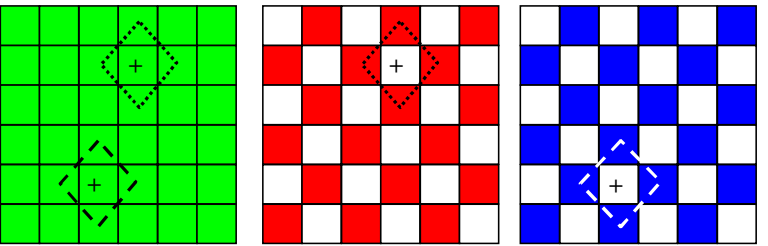

(d)
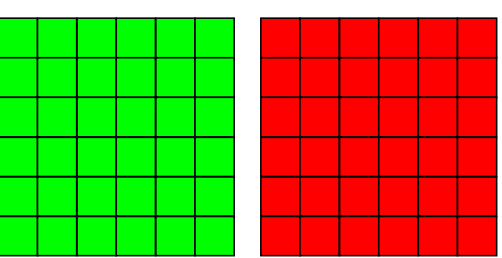

Fig. 4. Interpolation procedure based on the four-neighbor spatial arrangements: (a-c) incomplete color planes obtained during demosaicking (d) fully populated color planes of the demosaicked image. The widely used spatial arrangements of the available color components: (a,c) $\zeta=\{(r-1, s),(r, s-1),(r, s+1),(r+1, s)\}$, (b) $\zeta=\{(r-1, s-1)$, $(r-1, s+1),(r+1, s-1),(r+1, s+1)\}$.

located at the neighboring spatial locations $(r, s)$ and $(i, j)$ should exhibits the identical hue characteristics. It was argued in [8] that in smooth parts of the image the following hold:

$\frac{x_{(r, s) 1}}{x_{(i, j) 1}}=\frac{x_{(r, s) 2}}{x_{(i, j) 2}}$ or $\frac{x_{(r, s) 3}}{x_{(i, j) 3}}=\frac{x_{(r, s) 2}}{x_{(i, j) 2}}$

Based on (5), the interpolated value of the $\mathrm{R}(k=1)$ or $\mathrm{B}$ $(k=3)$ component $x_{(r, s) k}$ is obtained as:

$x_{(r, s) k}=x_{(r, s) 2} \frac{x_{(i, j) k}}{x_{(i, j) 2}}$

and analogously, the available $\mathrm{R}$ or B components can be used to obtain the $\mathrm{G}$ component as follows:

$x_{(r, s) 2}=x_{(r, s) k} \frac{x_{(i, j) 2}}{x_{(i, j) k}}$

Since natural color images do not have large areas with uniform hue characteristics, interpolation schemes of [8]-[13] smooth the color-ratios available in the spatial neighborhood ऽ. In this way, R (or B) components are obtained via :

$x_{(r, s) k}=x_{(r, s) 2} \sum_{(i, j) \in \varsigma} w_{(i, j)}^{\prime} \frac{x_{(i, j) k}}{x_{(i, j) 2}}$

where $x_{(r, s) 2}$ denotes the available $\mathrm{G}$ components placed at an interpolated location $(r, s)$, the quantities $x_{(i, j) k} / x_{(i, j) 2}$ denote the $\mathrm{R} / \mathrm{G}$ (for $k=1$ ) or $\mathrm{B} / \mathrm{G}$ (for $k=3$ ) color-ratios corresponding to the spatial location $(i, j) \in \varsigma$, which forms now a square shape mask $\zeta=\{(r-1, s-1),(r-1, s+1)$, $(r+1, s-1),(r+1, s+1)\}$, Fig. 4b. Since this interpolation step does not produce all needed R or B components, (8) should be repeated with the color-ratios located in a diamond-shaped (Fig. 4c) neighborhood $\varsigma=\{(r-1, s),(r, s-1),(r, s+1),(r+1, s)\}$. Then, the procedure results in fully populated $\mathrm{R}$ and $\mathrm{B}$ color planes (Fig. 4d).

In the case of the KA scheme [9], CFA zooming [12] and demosaicked image postprocessing [13] schemes, R or B color planes completed using (8) are used along with the original $G$ CFA components to re-evaluate $G$ components previously obtained without the use of the spectral information in (2). The interpolator determines the $\mathrm{G}$ component $x_{(r, s) 2}$ via:

$x_{(r, s) 2}=x_{(r, s) k} \sum_{(i, j) \in \varsigma} w_{(i, j)}^{\prime} \frac{x_{(i, j) 2}}{x_{(i, j) k}}$

where $x_{(i, j) 2} / x_{(i, j) k}$, for $(i, j) \in \varsigma$ and $\varsigma=\{(r-1, s),(r, s-1),(r, s+1)$, $(r+1, s)\}$, denotes $\mathrm{G} / \mathrm{R}$ (for $k=1$ ) or $\mathrm{G} / \mathrm{B}$ (for $k=3$ ) ratios. If an interpolated location $(r, s)$ corresponds to the B CFA location, then $k=1$ is used. Otherwise, $(r, s)$ corresponds to the R CFA location and B components $(k=3)$ are used in (9).

The components $x_{(r, s) 2}$ in (8) and $x_{(r, s) k}$ in (9) are used to normalize the smoothed ratio quantities to the appropriate intensity range. Since these components, located at the center of the spatial neighborhood $\varsigma$, describe the structural content of the image, the normalizing operations impress the highfrequency portion of the image to the interpolator's output.

Since the color-ratio model employed in (8) or (9) is based on the assumption of hue uniformity within a localized image area, it fails near edge transitions where both the spectral and spatial correlation characteristics of the image vary significantly [13]. As a result, the color-ratio based CFA interpolation schemes produce color artifacts. Moreover, the calculations of the color-ratios often results in singularities corresponding to strong color artifacts in the demosaicked image [13]. To avoid the aforementioned drawbacks, an alternative solution is needed.

\section{DEMOSAICKING USING NORMALIZED COLOR-RATIOS}

To overcome the problem, a normalization procedure for color-ratio inputs is introduced. The proposed here 
demosaicking operations improve the model's characteristics near the edge transitions while preserving the performance in uniform image areas. Note that such a normalization procedure can be realized in many ways. To provide a cost-effective solution which can be easily implemented in either hardware or software, two new color-ratio models are introduced: i) the first solution uses linear shifting of the inputs, and ii) the second design combines linear scaling and shifting operations.

\section{A. Linear shifting of the color-ratio inputs}

Let $\beta$ denote a non-negative shift parameter projecting the color components $x_{(r, s) k}$ to $x_{(r, s) k}+\beta$ and $x_{(i, j) k}$ to $x_{(i, j) k}+\beta$, for $k=1,2,3$. Thus, the underlying color-ratio model (5) changes to

$$
\frac{x_{(r, s) 1}+\beta}{x_{(i, j) 1}+\beta}=\frac{x_{(r, s) 2}+\beta}{x_{(i, j) 2}+\beta} \text { or } \frac{x_{(r, s) 3}+\beta}{x_{(i, j) 3}+\beta}=\frac{x_{(r, s) 2}+\beta}{x_{(i, j) 2}+\beta}
$$

respectively. This suggests that the proposed model generalizes (for $\beta=0$ ) the conventional color-ratio model of [8]. Note that the shift $\beta$ can be expressed as either a nonnegative constant or a positive-definite function of the color components inside the localized image area of support. With respect to the simplicity of the approach, we make use of a non-negative constant $\beta$.

Simple inspection reveals that in uniform image areas for any arbitrary value of $\beta$ the normalized ratio $\left(x_{(r, s) k}+\beta\right) /\left(x_{(i, j) k}+\beta\right) \quad$ is qualitatively identical to the conventional ratio $x_{(r, s) k} / x_{(i, j) k}$. However, near edge transitions the scale shift introduced in the normalized color-ratio model preserves the basic design philosophy of the interpolator while the conventional color-ratio model fails introducing thus shifted colors [13]. Under the new model the unknown R (for $k=1$ ) or $\mathrm{B}$ (for $k=3$ ) component at an interpolation location is calculated as

$$
x_{(r, s) k}=-\beta+\left(x_{(r, s) 2}+\beta\right) \frac{x_{(i, j) k}+\beta}{x_{(i, j) 2}+\beta}
$$

and analogously to this expression, the unknown $\mathrm{G}$ component is derived via

$$
x_{(r, s) 2}=-\beta+\left(x_{(r, s) k}+\beta\right) \frac{x_{(i, j) 2}+\beta}{x_{(i, j) k}+\beta}
$$

It should be noted that the interpolator's precision in estimating the magnitude of the color components increases with $\beta$. For example, in image areas with strong edges or fine details the relationship $c_{1} \neq c_{2} \neq c_{3}$ for $x_{(r, s) k} / x_{(i, j) k}=c_{k}$, is hampering the applicability of the conventional color-ratio model of [8]. On the contrary the linear scale shifting $\beta$ maps $\left(x_{(r, s) k}+\beta\right) /\left(x_{(i, j) k}+\beta\right)$ closer to unity, enforcing the underlying modeling assumption for both uniform and detail-rich areas. Since the procedure may shift large, in magnitude, color components beyond the upper limit of the conventionally used 8 -bit representation, a reasonable value for the parameter $\beta$ is $\beta=256$. Such a value constrains the normalized color components during processing within a 9-bit processing range. Straightforward addition of $-\beta$ to the intermediate result equivalent to $x_{(r, s) k}+\beta$ in (11) or $x_{(r, s) 2}+\beta$ in (12) maps the interpolated value back into the regular 8 -bit range.

Application of the normalized color-ratio model to the CFA interpolation schemes is relatively straightforward. To ensure the smooth normalized ratio characteristics in the localized image area $\varsigma$, the normalized ratio quantities are used in the inputs of the interpolator averaging in nature. Thus, instead of the conventional definition (8), the $\mathrm{R}$ (for $k=1$ ) or $\mathrm{B}$ (for $k=3$ ) component $x_{(r, s) k}$ is obtained as follows:

$x_{(r, s) k}=-\beta+\left(x_{(r, s) 2}+\beta\right) \sum_{(i, j) \in \varsigma} w_{(i, j)}^{\prime} \frac{x_{(i, j) k}+\beta}{x_{(i, j) 2}+\beta}$

where $w_{(i, j)}^{\prime}$ is the normalized edge-sensing coefficient which regulates the contribution of the normalized $\mathrm{R} / \mathrm{G}$ or $\mathrm{B} / \mathrm{G}$ ratios $\left(x_{(i, j) k}+\beta\right) /\left(x_{(i, j) 2}+\beta\right)$ to the interpolated output $x_{(r, s) k}$. The normalized $\mathrm{G}$ component $\left(x_{(r, s) 2}+\beta\right)$ and the parameter $-\beta$ are used to map the normalized color-ratio interpolator's output to the pixel domain. Assuming a KA-style interpolator [9], a color-ratio based digital camera pipeline of [12], or a post-processing scheme of [13], which utilize the G/R and $\mathrm{G} / \mathrm{B}$ ratios in the re-interpolating of the $\mathrm{G}$ components, the interpolator's $\mathrm{G}$ output should be obtained via:

$x_{(r, s) 2}=-\beta+\left(x_{(r, s) k}+\beta\right) \sum_{(i, j) \in \varsigma} w_{(i, j)}^{\prime} \frac{x_{(i, j) 2}+\beta}{x_{(i, j) k}+\beta}$

Note that the area of support $\varsigma$ as well as the weight calculation may be appropriately modified depending on the underlying CFA pattern and demosaicking scheme employed.

\section{B. Scaling and shifting operations on the color-ratio inputs}

The second solution is based on a linear transformation utilized in color image enhancement [17]. The procedure can be seen as a combination of scaling and shifting operations. It is claimed in [17] that such an operation can be designed to preserve the hue characteristics of the image.

Let $\alpha$ be a positive scaling factor and $\beta$ denote a shift parameter. The procedure normalizes the color components $x_{(r, s) k}$ to $\alpha x_{(r, s) k}+\beta$ and $x_{(i, j) k}$ to $\alpha x_{(i, j) k}+\beta$, for $k=1,2,3$. Thus, the underlying color-ratio model (5) changes to the following expression:

$$
\frac{\alpha x_{(r, s) 1}+\beta}{\alpha x_{(i, j) 1}+\beta}=\frac{\alpha x_{(r, s) 2}+\beta}{\alpha x_{(i, j) 2}+\beta} \text { or } \frac{\alpha x_{(r, s) 3}+\beta}{\alpha x_{(i, j) 3}+\beta}=\frac{\alpha x_{(r, s) 2}+\beta}{\alpha x_{(i, j) 2}+\beta}
$$

respectively. It is not difficult to see that for $\alpha=1$ and $\beta=0$ the proposed normalized color-ratio model generalizes the 


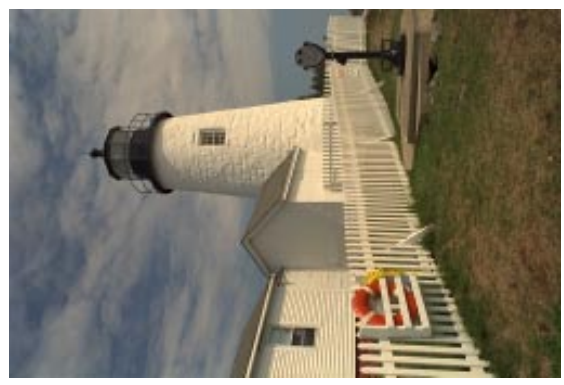

(a)

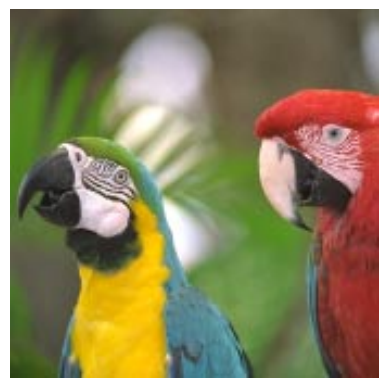

(b)

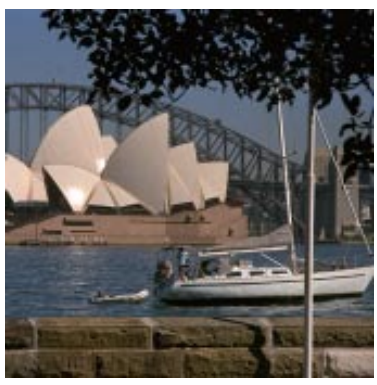

(c)

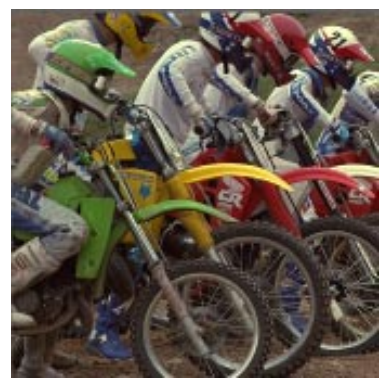

(d)

Fig. 5. Test color images: (a) Lighthouse, (b) Parrots, (c) Sydney, (d) Bikes.

conventional color-ratio model of [8], and for $\alpha=1$ the proposed model (15) generalizes the linear-shifting based model defined in (10).

Under the new model the $\mathrm{R}(k=1)$ or $\mathrm{B}(k=3)$ components $x_{(r, s) k}$ at an interpolation location $(r, s)$ are calculated using:

$x_{(r, s) k}=\frac{1}{\alpha}\left[-\beta+\left(\alpha x_{(r, s) 2}+\beta\right) \frac{\alpha x_{(i, j) k}+\beta}{\alpha x_{(i, j) 2}+\beta}\right]$

On the other hand, the G component $x_{(r, s) 2}$ is obtained via

$x_{(r, s) 2}=\frac{1}{\alpha}\left[-\beta+\left(\alpha x_{(r, s) k}+\beta\right) \frac{\alpha x_{(i, j) 2}+\beta}{\alpha x_{(i, j) k}+\beta}\right]$

Similarly as in the shifted version of the model, the linear transformation used in (16) and (17) normalizes the dynamic range of the color-ratios. At the same time, it makes the underlying modeling assumption valid in both uniform and detail-rich areas.

To ensure the smooth characteristics of the interpolated image, the CFA interpolator averages the normalized ratios corresponding to spatially neighboring locations $(i, j) \in \varsigma$. Therefore, the $\mathrm{R}$ or $\mathrm{B}$ component $x_{(r, s) k}$ is determined as follows:

$x_{(r, s) k}=\frac{1}{\alpha}\left[-\beta+\left(\alpha x_{(r, s) 2}+\beta\right) \sum_{(i, j) \in \zeta} w_{(i, j)}^{\prime} \frac{\alpha x_{(i, j) k}+\beta}{\alpha x_{(i, j) 2}+\beta}\right]$

while the $\mathrm{G}$ component is outputted using

$x_{(r, s) 2}=\frac{1}{\alpha}\left[-\beta+\left(\alpha x_{(r, s) k}+\beta\right) \sum_{(i, j) \in \zeta} w_{(i, j)}^{\prime} \frac{\alpha x_{(i, j) 2}+\beta}{\alpha x_{(i, j) k}+\beta}\right]$

In the above equations $\varsigma$ denotes the area of support. The normalized components $\left(\alpha x_{(r, s) 2}+\beta\right)$ in (18) and $\left(\alpha x_{(r, s) k}+\beta\right)$ in (19) are used to normalize the operand of the weighted average operator from the ratio to normalized (scaled and shifted) intensity domain. To recover the original intensities, the addition of $-\beta$ ensures inverse shifting normalization of the outputted values and the procedure completes with inverse scaling normalization realized through the use of an $1 / \alpha$ normalization factor. Note that the proposed normalized models (10) and (15) enforce the hue uniformity considered as the underlying modeling assumption in a robust way. Although the models normalize discontinuities in the intensity to ensure the correct utilization of the spectral information, the interpolators weights $w_{(i, j)}$ are used to follow the edge information. It will be shown that combining both the normalized color-ratio model and the edge sensing mechanism, the KA and SAI schemes produce excellent improvements compared against the case when the these interpolators use conventional color-ratios.

\section{Other (nonlinear) alternatives}

Due to a nonlinear nature of the image [18], a nonlinear transformation of the local image characteristics may be a better alternative than the previous two linear models. It has been argued that nonlinear transformations can be designed to preserve the hue characteristics [17].

A general transformation changes the inputs $x_{(r, s) k}$ to $\alpha\left(x_{(r, s) k}\right) x_{(r, s) k}+\beta\left(x_{(r, s) k}\right)$ and $x_{(i, j) k}$ to $\alpha\left(x_{(i, j) k}\right) x_{(i, j) k}+\beta\left(x_{(i, j) k}\right)$, for $k=1,2,3$. This generalized transformations is based on $\alpha($.) and $\beta($.$) denoting any two functions defined over the$ corresponding inputs $x_{(r, s) k}$ or $x_{(i, j) k}$, [17]. Although such a procedure can produce excellent results, its high computational complexity and difficulties with a setting of de-normalizing nonlinearities limit the applicability of the nonlinear color-ratio modeling in demosaicking. Therefore, we use the linear shifting based (13),(14) and the linear shifting/scaling based (18),(19) throughout the paper.

\section{EXPERIMENTAL RESULTS}

The color images shown in Fig. 5 have been used to evaluate the proposed models. The test images, captured using three-sensor devices, have been normalized to 8-bit per channel RGB representation. Except the image Lighthouse (Fig. 5a) which is $768 \times 512$ in size, other images such as Parrots (Fig. 5b), Sydney (Fig. 5c) and Bikes (Fig. 5d) are square $512 \times 512$ images. Note that these images contain image regions usually problematic for CFA interpolation schemes which produce undesired side-effects in the form of aliasing noise, color shifts, and zipper effects [4],[5],[13],[15],[19]. 
TABLE I

COMPARISON OF THE METHODS USING THE LIGHTHOUSE IMAGE

\begin{tabular}{crrr}
\hline \hline Method & MAE & MSE & NCD \\
\hline BI & 4.468 & 108.3 & 0.0653 \\
MFI & 2.341 & 31.8 & 0.0380 \\
EMI & 2.322 & 23.1 & 0.0370 \\
API & 1.817 & 12.7 & 0.0298 \\
C2D2 & 2.005 & 16.2 & 0.0301 \\
PVM & 2.364 & 23.5 & 0.0366 \\
BD & 2.149 & 19.7 & 0.0321 \\
standard SHT & 3.619 & 58.5 & 0.0522 \\
standard KA & 2.241 & 19.4 & 0.0365 \\
standard SAI & 2.570 & 24.3 & 0.0403 \\
SHT (13) & 3.398 & 53.8 & 0.0489 \\
KA (13),(14) & 1.572 & 9.2 & 0.0247 \\
SAI (13) & 2.142 & 18.9 & 0.0329 \\
SHT (18) & 3.390 & 53.6 & 0.0489 \\
KA (18),(19) & 1.556 & 8.9 & 0.0243 \\
SAI (18) & 2.120 & 18.4 & 0.0324 \\
\hline
\end{tabular}

TABLE II

COMPARISON OF THE METHODS USING THE PARROTS IMAGE

\begin{tabular}{crrr}
\hline \hline Method & MAE & MSE & NCD \\
\hline BI & 2.128 & 31.4 & 0.0262 \\
MFI & 1.198 & 7.2 & 0.0172 \\
EMI & 1.598 & 16.9 & 0.0207 \\
API & 1.238 & 6.2 & 0.0173 \\
C2D2 & 1.216 & 6.2 & 0.0163 \\
PVM & 1.531 & 11.8 & 0.0199 \\
BD & 1.202 & 6.0 & 0.0170 \\
standard SHT & 1.925 & 18.8 & 0.0245 \\
standard KA & 2.688 & 94.6 & 0.0357 \\
standard SAI & 1.739 & 12.1 & 0.0224 \\
SHT (13) & 1.671 & 15.7 & 0.0215 \\
KA (13),(14) & 1.180 & 5.6 & 0.0162 \\
SAI (13) & 1.337 & 8.7 & 0.0176 \\
SHT (18) & 1.664 & 15.5 & 0.0215 \\
KA (18),(19) & 1.159 & 5.2 & 0.0160 \\
SAI (18) & 1.331 & 8.6 & 0.0175 \\
\hline
\end{tabular}

SHT, KA and SAI demosaicking schemes operating on the proposed normalized color-ratios are compared, in terms of performance, against the conventional color-ratio model based SHT, KA and SAI schemes. Note that SHT and SAI use the spectral information to interpolate R or B components. Therefore, the new variants of these schemes can utilize (13) or (18), only. On the other hand, the new KA variants use the spectral characteristics in (13),(14) or (18),(19) to produce the full color output. Experimentation with a wide range of color images showed that (13) and (14) should be used with $\beta=256$. Note that this value of $\beta$ remains the inputs within the 9-bit processing range. On the other hand, $\alpha=0.05$ and $\beta=256$ were found to boost performance of (18) and (19). To illustrate improvements of SHT, $\mathrm{KA}$, and SAI schemes corresponding with the use of the proposed normalization procedures, the methods are compared against stateof-the-art demosaicking schemes such as the bilinear interpolation scheme [19],[20], the median filter interpolation (MFI) scheme [21], the edge map interpolation (EMI) scheme [22], the adaptive color plane interpolation (API) scheme [23], the color correlationdirectional derivatives (C2D2) scheme [4], the principle vector method (PVM) [5], and the bilinear difference (BD) interpolation scheme [15].
TABLE III

COMPARISON OF THE METHODS USING THE SYDNEY IMAGE

\begin{tabular}{crrr}
\hline Method & MAE & MSE & NCD \\
\hline BI & 4.792 & 99.0 & 0.1095 \\
MFI & 2.276 & 22.2 & 0.0616 \\
EMI & 2.318 & 21.7 & 0.0584 \\
API & 1.895 & 13.7 & 0.0529 \\
C2D2 & 1.968 & 15.0 & 0.0485 \\
PVM & 2.595 & 28.8 & 0.0650 \\
BD & 2.180 & 16.1 & 0.0625 \\
standard SHT & 3.672 & 51.7 & 0.0861 \\
standard KA & 2.295 & 22.7 & 0.0651 \\
standard SAI & 2.470 & 23.0 & 0.0641 \\
SHT (13) & 3.501 & 47.9 & 0.0820 \\
KA (13),(14) & 1.494 & 8.0 & 0.0388 \\
SAI (13) & 2.151 & 19.3 & 0.0519 \\
SHT (18) & 3.454 & 47.0 & 0.0846 \\
KA (18),(19) & 1.499 & 8.0 & 0.0397 \\
SAI (18) & 2.159 & 19.7 & 0.0531 \\
\hline
\end{tabular}

TABLE IV

COMPARISON OF THE METHODS USING THE BIKES IMAGE

\begin{tabular}{cccc}
\hline \hline Method & MAE & MSE & NCD \\
\hline BI & 6.117 & 157.1 & 0.1209 \\
MFI & 2.540 & 27.4 & 0.0620 \\
EMI & 3.332 & 58.8 & 0.0744 \\
API & 2.335 & 22.6 & 0.0551 \\
C2D2 & 2.481 & 26.7 & 0.0545 \\
PVM & 3.644 & 59.0 & 0.0791 \\
BD & 2.396 & 20.5 & 0.0581 \\
standard SHT & 4.842 & 94.8 & 0.1025 \\
standard KA & 3.410 & 71.7 & 0.0792 \\
standard SAI & 3.375 & 53.1 & 0.0768 \\
SHT (13) & 4.530 & 82.6 & 0.0965 \\
KA (13),(14) & 2.094 & 17.2 & 0.0489 \\
SAI (13) & 2.910 & 40.9 & 0.0629 \\
SHT (18) & 4.537 & 82.6 & 0.0975 \\
KA (18),(19) & 2.090 & 17.0 & 0.0489 \\
SAI (18) & 2.907 & 40.7 & 0.0629 \\
\hline
\end{tabular}

Following common practices in the research community, mosaic versions of the images are created by discarding color information in a GRGR phased Bayer CFA filter [5],[13],[14]. Demosaicked images are generated using each of the listed methods. The efficiency of the interpolation methods is measured, objectively, via the mean absolute error (MAE), the mean square error (MSE) and the normalized color difference (NCD) criterion [7],[18].

Tables I-IV summarize the results obtained using the test images shown in Fig. 5. It can be seen that standard SHT, KA, SAI schemes, which operate on the conventional color-ratio model of (5) produce worse results compared to API, C2D2 or BD schemes. However, if the simple normalized color-ratio models are employed, impressive improvements produced by SHT, KA and SAI are noticed. This is most significant for the KA which utilizes an iterative correction cycle. These results indicate that depending on the edge sensing mechanism and the interpolation/correction steps employed the use of the normalized color-ratio models allows to design powerful demosaicking tools. Moreover, the proposed models can be easily implemented in either software or hardware. 


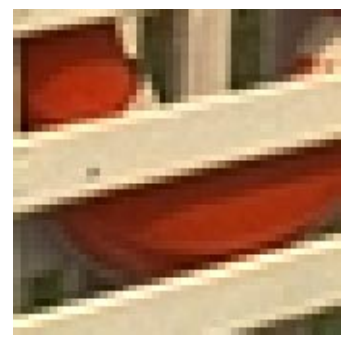

(a)

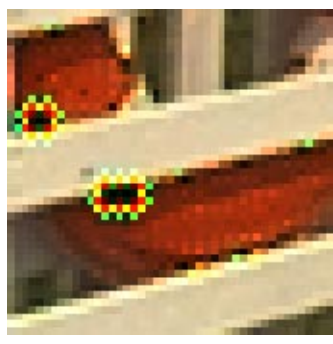

(b)

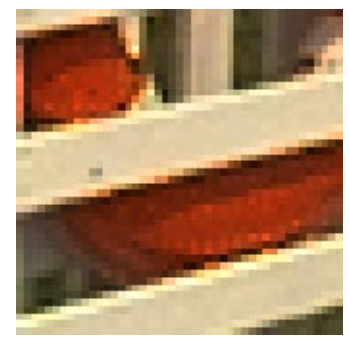

(c)

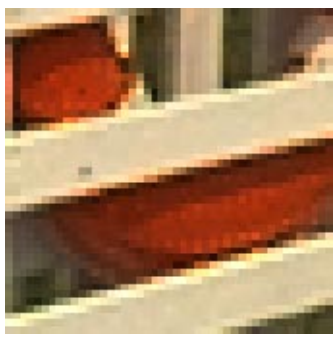

(d)

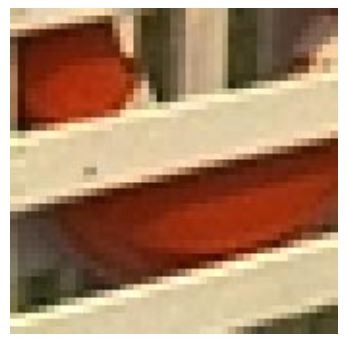

(e)

Fig. 6. Enlarged parts of the original Lighthouse image (a) and the output images (b-e) obtained using the KA scheme operating on (13) and (14) with: (b) $\beta=0$, (c) $\beta=5$, (d) $\beta=10$, (e) $\beta=256$.

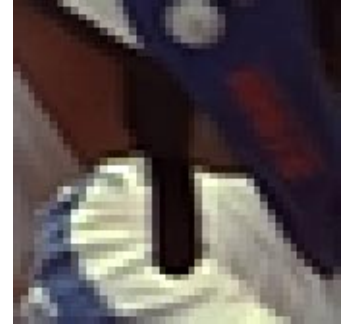

(a)

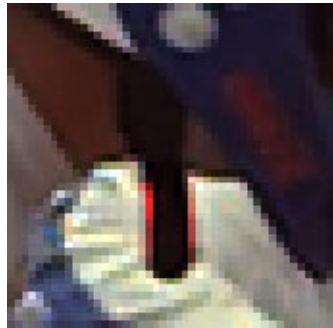

(b)

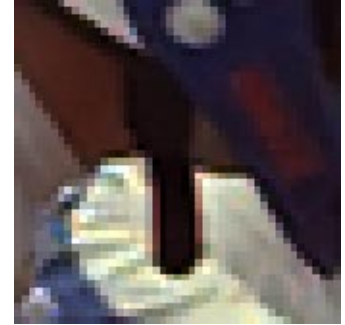

(c)

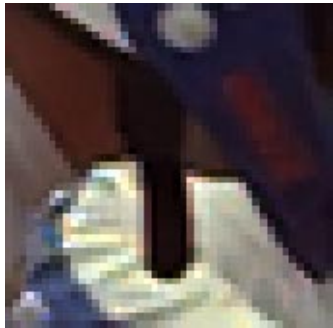

(d)

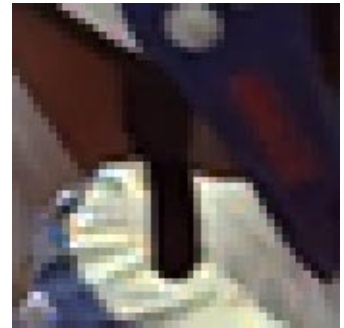

(e)

Fig. 7. Enlarged parts of the original Bikes image (a) and the output images (b-e) obtained using the SAI scheme operating on (13) with: (b) $\beta=0$, (c) $\beta=5$, (d) $\beta=10$, (e) $\beta=256$.

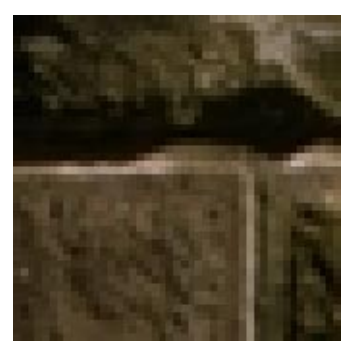

(a)

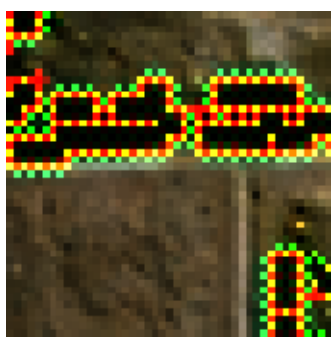

(b)

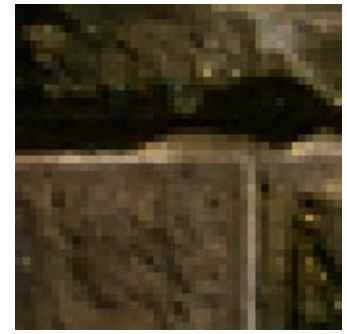

(c)

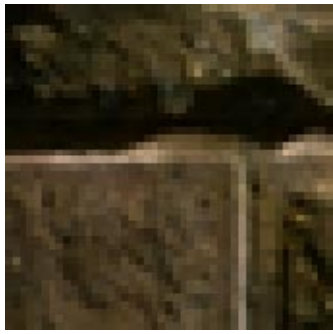

(d)

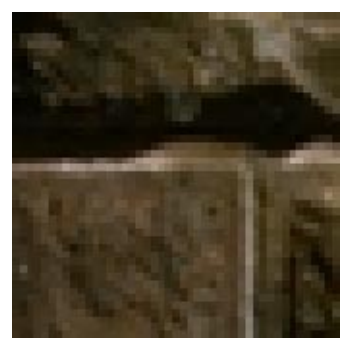

(e)

Fig. 8. Enlarged parts of the original Sydney image (a) and the output images (b-e) obtained using the KA scheme operating on (18) and (19) with: (b) $\alpha=1$ and $\beta=0$, (c) $\alpha=1$ and $\beta=5$, (d) $\alpha=0.5$ and $\beta=10$, (e) $\alpha=0.05$ and $\beta=256$.

Fig. 6 depicts the outputs obtained using the test image Lighthouse and the KA scheme operating on the proposed linear shifting based color-ratio model (13) and (14). Fig. 6b shows that the use of $\beta=0$ causes singularities in the ratio calculations resulting in strong color artifacts. The small increase in $\beta$ eliminates this problem, however, color shifts are still present in the demosaicked outputs (Figs. 6c,d). Visual inspection of the original image (Fig. 6a) and the demosaicked output shown in Fig. 6e reveals that the use of the $\beta=256$ results in the naturally colored images without the presence of color shifts and artifacts. The similar behavior is observed when the SAI scheme and the linearly shifting model (13) are used (Fig. 7). The scheme avoids color shifts (Figs. 7b-d) if $\beta=256$ is employed in the model (Fig. 7e). Fig. 8 depicts the results corresponding to the KA scheme operating on the proposed shifting/scaling model (18) and (19). Similarly as in the previous cases, the use of $\alpha=0.05$ and $\beta=256$ is not accompanied (Fig. 8e) with the observation of the undesired-side effects in the low-intensity regions. At the same time, the scheme produces the output with the highest fidelity to the original (Fig. 8a).
Fig. 9 depicts enlarged parts of the test images cropped in edge areas which are usually problematic for demosaicking schemes. These results allow for the visual comparison of the methods considered here. It can be seen that the use of BI, MFI, EMI, SAI, KA results in color shits (Figs. 8b-d,g,i). On the other hand, C2D2 and API produce better results (Figs. 8e,f), however, the use of the KA scheme (Fig. 8j) operating on the proposed color-model (13) and (14) outperforms these methods producing the best image quality.

In the summary, the following conclusions can be drawn: i) the use of the proposed normalized models (13),(14) or (18),(19) results in visually pleasing color outputs, ii) the color-ratio inputs should be transformed close to the unity enforcing the underlying modeling assumptions in both high-frequency and smooth image regions, iii) the use of the recommended settings $\beta=256$ in (13),(14) and $\alpha=0.05, \quad \beta=256$ in (18),(19) avoids color-shifts and aliasing artifacts in the demosaicked outputs, iv) operating on the proposed models (13),(14) or (18),(19) the KA becomes one of the most powerful demosaicking schemes, and v) the proposed models can be easily implemented either in software or hardware. 


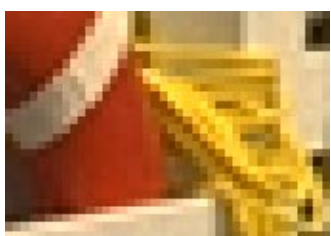

(a1)

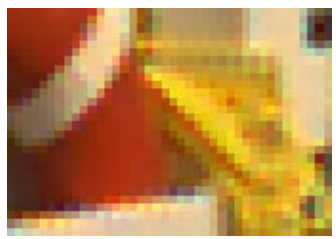

(b1)

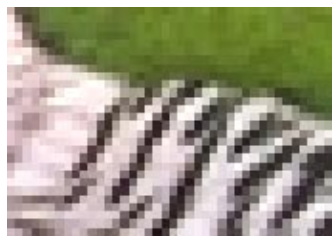

(a2)

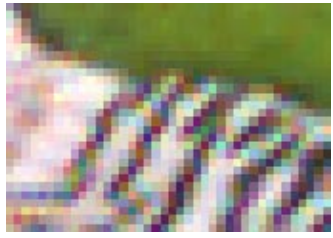

(b2)

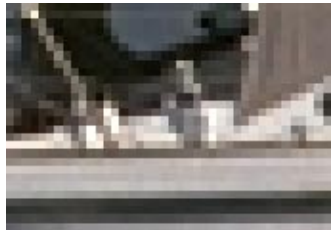

(a3)

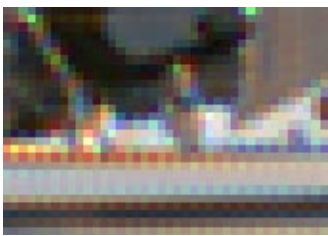

(b3)

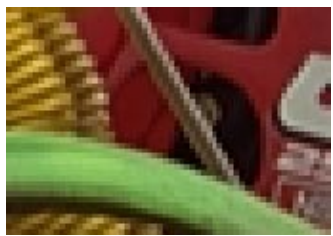

(a4)

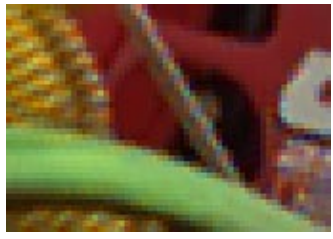

(b4)

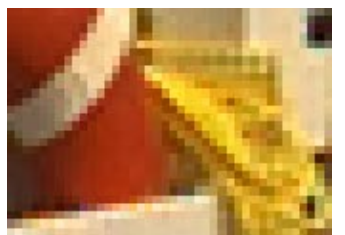

(c1)

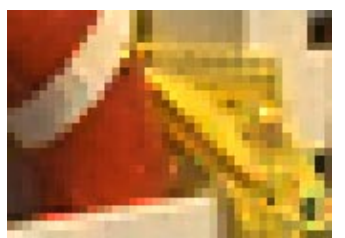

(d1)

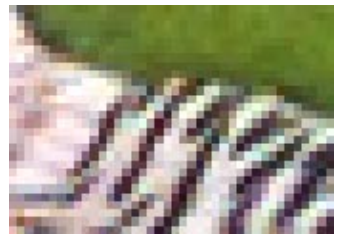

(c2)

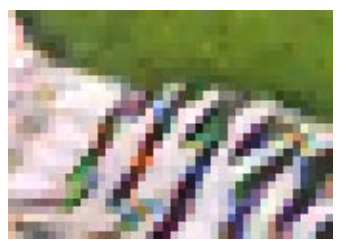

(d2)

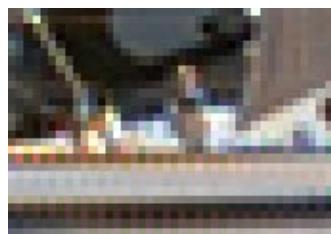

(c3)

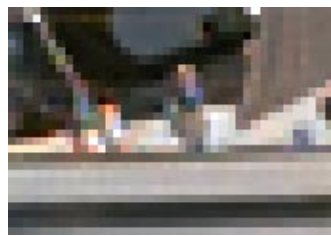

(d3)

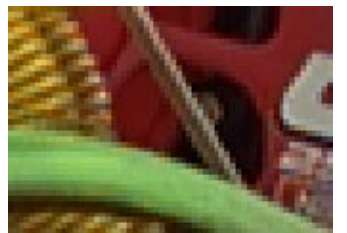

(c4)

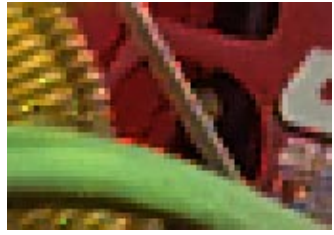

(d4)

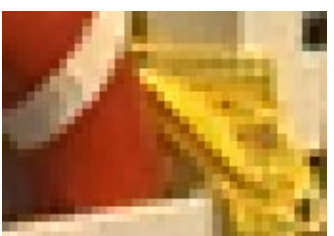

(e1)

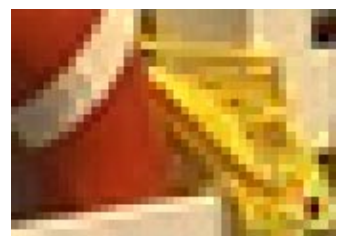

(f1)

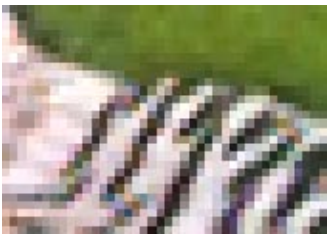

(e2)

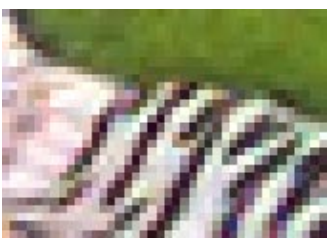

(f2)

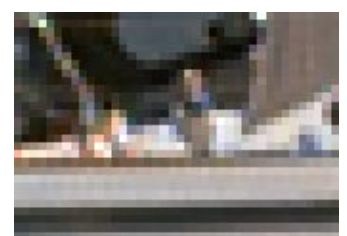

(e3)

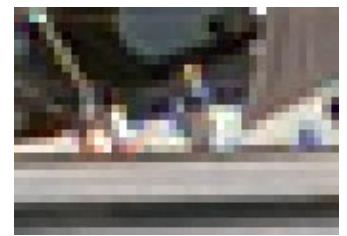

(f3)

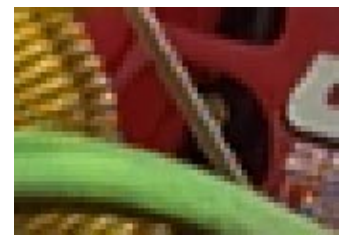

(e4)

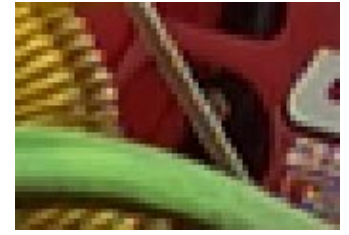

(f4)

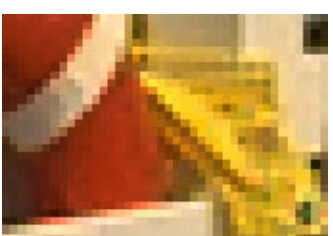

(g1)

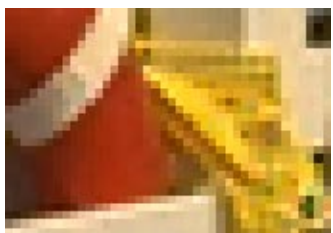

(h1)

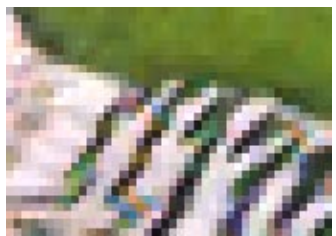

(g2)

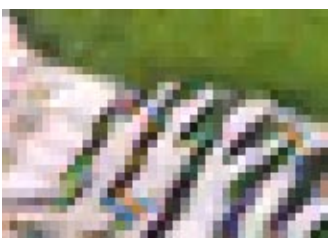

(h2)

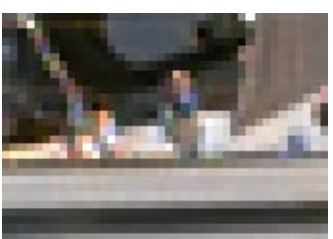

(g3)

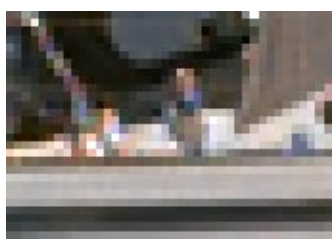

(h3)

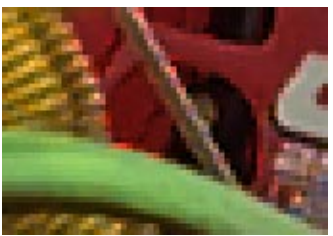

(g4)

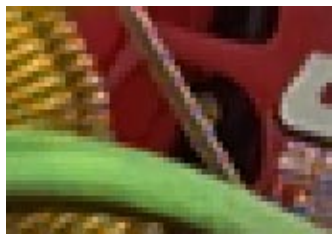

(h4)

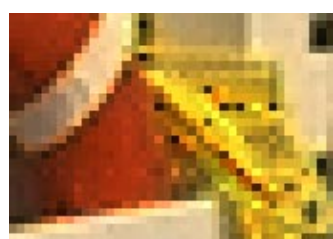

(i1)

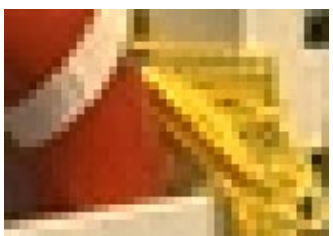

(j1)

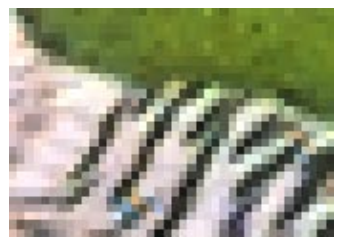

(i2)

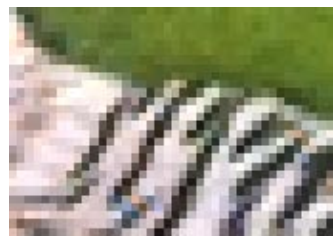

(j2)

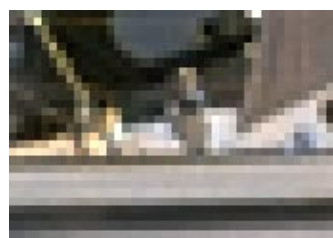

(i3)

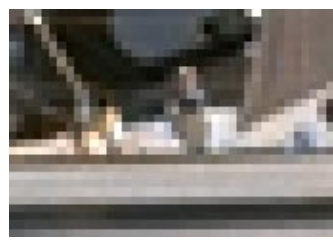

(j3)

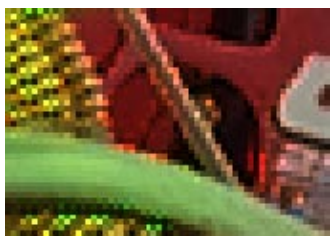

(i4)

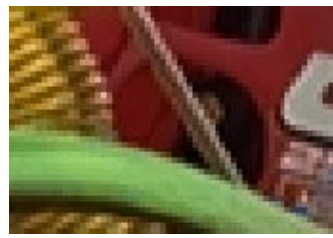

(j4)

Fig. 9. Enlarged parts of the images: (1) Lighthouse, (2) Parrots, (3) Sydney, (4) Bikes. The achieved results correspond to: (a) original image, (b) BI output, (c) MFI output, (d) EMI output, (e) C2D2 output, (f) API output, (g) standard SAI output, (h) SAI output obtained using (13) with $\beta=256$, (i) standard KA output, (j) KA output obtained using (13) and (14) with $\beta=256$. 


\section{CONClusion}

A normalized color-ratio model for single-sensor camera image processing was introduced. The first solution utilizes linear shifts to alleviate effects of edge variations in the interpolator's input. The second solution take advantages of both the linear scaling and shifting operations to normalize the color-ratio variations in the interpolator's input. Employing the proposed model instead of the conventional color-ratio model along with typical CFA interpolation procedures, excellent demosaicking results can be obtained.

\section{ACKNOWLEDGMENT}

The work of the first author is supported by a NATO/NSERC Science award.

\section{REFERENCES}

[1] B.E. Bayer, "Color imaging array," U.S. Patent 3971 065, 1976.

[2] J. Adams, K. Parulski, and K. Spaulding, "Color processing in digital cameras," IEEE Micro, vol. 18, no. 6, pp. 20-30, Nov.-Dec. 1998.

[3] J. Adams, "Design of practical color filter array interpolation algorithms for digital cameras," Proceedings of the SPIE, vol. 3028, pp. 117-125, February 1997.

[4] N. Kehtarnavaz, H.J Oh, and Y. Yoo, "Color filter array interpolation using color correlations and directional derivatives," Journal of Electronic Imaging, vol. 12, no. 4, pp. 621-632, October 2003.

[5] R. Kakarala and Z. Baharav, "Adaptive demosaicing with the principal vector method," IEEE Transactions on Consumer Electronics, vol. 48, pp. 932-937, November 2002.

[6] H.J. Trussell and R.E. Hartwig, "Mathematics for demosaicking," IEEE Transactions on Image Processing, vol. 11, no. 4, pp. 485-492, April 2002.

[7] R. Lukac, K.N. Plataniotis, D. Hatzinakos, and M. Aleksic, "A novel cost effective demosaicing approach," IEEE Transactions on Consumer Electronics, vol. 50, no. 1, February 2004.

[8] D.R. Cok, "Signal processing method and apparatus for producing interpolated chrominance values in a sampled color image signal," US Patent 4642 678, 1987.

[9] R. Kimmel, "Demosaicing: image reconstruction from color CCD samples," IEEE Transactions on Image Processing, vol. 8, pp. 12211228, September 1999.

[10] C. Cai, T.H. Yu, and S.K. Mitra, "Saturation-based adaptive inverse gradient interpolation for Bayer pattern images," IEE Proceedings Vision, Image, Signal Processing, vol. 148, no. 3, pp. 202-208, June 2001.

[11] R. Ramanath, W.E. Snyder, G.L. Bilbro, and W.A. Sander III, "Demosaicking methods for Bayer color arrays," Journal of Electronic Imaging, vol. 11, no. 3, pp. 306-315, July 2002.

[12] R. Lukac, K. Martin, and K.N. Plataniotis, "Digital camera zooming based on unified CFA image processing steps," IEEE Transactions on Consumer Electronics, vol. 50, no. 1, February 2004.

[13] R. Lukac, K. Martin, and K.N. Plataniotis, "Demosaicked image postprocessing using local color ratios," IEEE Transactions on Circuit and Systems for Video Technology, vol. 14, no. 6, June 2004.

[14] B. Gunturk, Y. Altunbasak, and R. Mersereau, "Color plane interpolation using alternating projections," IEEE Transactions on Image Processing, vol. 11, no.9, pp. 997-1013, September 2002.

[15] S.C. Pei and I.K. Tam, "Effective color interpolation in CCD color filter arrays using signal correlation," IEEE Trans. Circuits and Systems for Video Technology, vol. 13, no. 6, pp. 503-513, June 2003.

[16] R. Lukac and K.N. Plataniotis, "A new color restoration solution for Bayer pattern based imaging devices," IEEE Signal Processing Letters, Vol.11, to appear 2004.

[17] S.K. Naik and C.A. Murthy, "Hue-preserving color image enhancement without gamut problem," IEEE Transactions on Image Processing, vol. 12, pp. 1591-1598, December 2003.
[18] K.N. Plataniotis and A.N. Venetsanopoulos, Color Image Processing and Applications. Springer Verlag, Berlin, 2000.

[19] P. Longere, Z. Xuemei, P.B. Delahunt, and D.H. Brainard, "Perceptual assessment of demosaicing algorithm performance," Proceedings of the IEEE, vol. 90, no. 1, pp. 123-132, January 2002.

[20] T. Sakamoto, C. Nakanishi, and T. Hase, "Software pixel interpolation for digital still cameras suitable for a 32-bit MCU," IEEE Transactions on Consumer Electronics, vol. 44, no. 4, pp. 1342-1352, November 1998.

[21] W.T. Freeman, "Median filter for reconstructing missing color samples," U.S. Patent 5373 322, 1988.

[22] B.S. Hur and M.G. Kang, "High definition color interpolation scheme for progressive scan CCD image sensor," IEEE Trans. Consumer Electronics, vol. 47, no. 2, pp. 179-186, February 2001.

[23] J.F. Hamilton and J.E. Adams, "Adaptive color plane interpolation in single sensor color electronic camera," U.S. Patent 5 629 734, 1997.

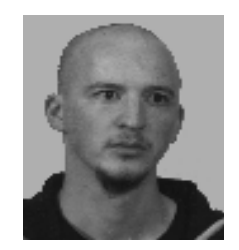

Rastislav Lukac received a Diploma in Telecommunications with honors in 1998 and a Ph.D. in 2001, both at the Technical University of Kosice, Slovak Republic. From February 2001 to August 2002 he was an assistant professor at the Department of Electronics and Multimedia Communications at the Technical University of Kosice. Since August 2002 he is a researcher in Slovak Image Processing Center in Dobsina, Slovak Republic. From January 2003 to March 2003 he was a postdoc at Artificial Intelligence \& Information Analysis Lab at the Aristotle University of Thessaloniki, Greece. In 2003, he was awarded the NATO Science Fellowship. Since May 2003 he has been a post-doctoral fellow at the Edward S. Rogers Sr. Department of Electrical and Computer Engineering at the University of Toronto in Toronto, Canada.

Dr. Lukac is a member of the IEEE Signal Processing Society. He is an active member of Review and Program Committees at various European conferences and a reviewer for various scientific journals. Recently, his research interests include nonlinear digital filters, image sharpening and analysis, color image processing, CFA interpolation/zooming and digital camera image processing, image sequence processing, multimedia, and the use of Boolean functions, permutation theory and artificial intelligence in filter design.

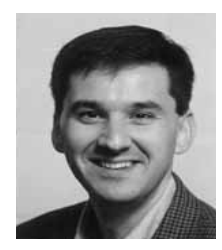

Konstantinos N. Plataniotis received the B. Engineering degree in Computer Engineering from the Department of Computer Engineering and Informatics, University of Patras, Patras, Greece in 1988 and the M.S and Ph.D degrees in Electrical Engineering from the Florida Institute of Technology (Florida Tech), Melbourne, Florida in 1992 and 1994 respectively. He was affiliated with the Computer Technology Institute (C.T.I), Patras, Greece from 1989 to 1991 . He was with the Digital Signal \& Image Processing Laboratory, Department of Electrical and Computer Engineering University of Toronto, from 1995 to 1997. From August 1997 to June 1999 he was an Assistant Professor with the School of Computer Science at Ryerson University. While at Ryerson Prof. Plataniotis served as a lecturer in 12 courses to industry and Continuing Education programs. Since 1999 he has been with the University of Toronto. He is currently an Assistant Professor at the Edward S. Rogers Sr. Department of Electrical \& Computer Engineering where he researches and teaches adaptive systems and multimedia signal processing.

Dr. Plataniotis is the Bell Canada Junior Chairholder in Multimedia and a Nortel Institute for Telecommunications Associate. He co-authored, with A.N. Venetsanopoulos, a book on "Color Image Processing \& Applications", Springer Verlag, May 2000, ISBN 3-540-66953-1, he is a contributor to four books, and he has published more than 200 papers in refereed journals and conference proceedings in the areas of multimedia signal processing, image processing, adaptive systems, communications systems and stochastic estimation.

Dr. Plataniotis is a Senior Member of IEEE, a past member of the IEEE Technical Committee on Neural Networks for Signal Processing, and the Technical Co-Chair of the Canadian Conference on Electrical and Computer Engineering, CCECE 2001, May 13-16, 2001, and CCECE 2004, May 2-5 2004. 